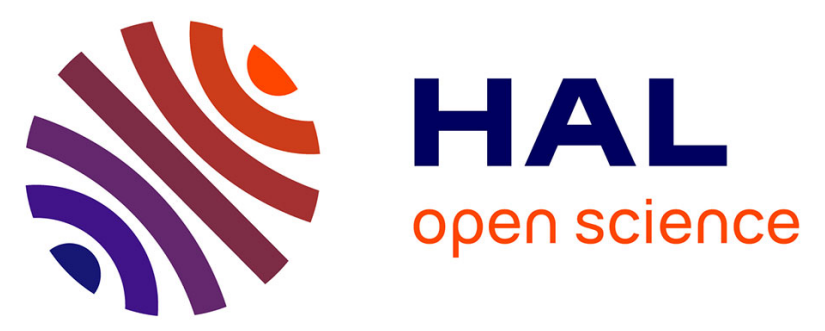

\title{
Investigation of the potentialities of Vertical Resistive RAM (VRRAM) for neuromorphic applications
}

\author{
G. Piccolboni, G. Molas, M. Portal, R. Coquand, Marc Bocquet, D. Garbin, \\ E. Vianello, C. Carabasse, V. Delaye, C. Pellissier, et al.
}

\section{- To cite this version:}

G. Piccolboni, G. Molas, M. Portal, R. Coquand, Marc Bocquet, et al.. Investigation of the potentialities of Vertical Resistive RAM (VRRAM) for neuromorphic applications. 2015 IEEE International Electron Devices Meeting (IEDM), Dec 2015, Washington, United States. pp.17.2.1-17.2.4, 10.1109/IEDM.2015.7409717 . hal-01804658

\section{HAL Id: hal-01804658 \\ https://hal.science/hal-01804658}

Submitted on 1 Jun 2018

HAL is a multi-disciplinary open access archive for the deposit and dissemination of scientific research documents, whether they are published or not. The documents may come from teaching and research institutions in France or abroad, or from public or private research centers.
L'archive ouverte pluridisciplinaire HAL, est destinée au dépôt et à la diffusion de documents scientifiques de niveau recherche, publiés ou non, émanant des établissements d'enseignement et de recherche français ou étrangers, des laboratoires publics ou privés. 


\title{
Investigation of the potentialities of Vertical Resistive RAM (VRRAM) for neuromorphic applications
}

G. Piccolboni, G. Molas, J. M. Portal ${ }^{1}$, R. Coquand, M. Bocquet ${ }^{1}$, D. Garbin, E. Vianello, C. Carabasse, V. Delaye, C. Pellissier, T. Magis, C. Cagli, M. Gely, O. Cueto, D. Deleruyelle$^{1}$, G. Ghibaudo², B. De Salvo, L. Perniola

\author{
CEA, LETI, MINATEC Campus, GRENOBLE, France, giuseppe.piccolboni@cea.fr, (1) IM2NP Marseille, France, (2) IMEP LAHC CNRS Grenoble, France
}

\section{Introduction}

Combining Resistive RAM concept with Vertical NAND technology and design, Vertical RRAM (VRRAM) was recently proposed as a cost-effective and extensible technology for future mass data storage applications [1]. 3D RRAM based neural networks were also proposed to emulate the potentiation and depression of a synapse [2], but more complex circuits were not discussed. In previous works [3-4], various RRAM based neuromorphic circuits were proposed and investigated, using planar devices.

In this paper, we investigate for the $1^{\text {st }}$ time the potentiality of the VRRAM concept for various neuromorphic applications, one synapse being emulated by one VRRAM pillar. First, basic functionality of $\mathrm{HfO}_{2}$ based VRRAM is presented. 20ns switching time, up to $10^{7}$ cycles and stable $200^{\circ} \mathrm{C}$ retention were demonstrated. Then specific analyses are made on the resistance and switching voltage variability. We demonstrate that a correlation effect exists between adjacent cycles, meaning the filament keeps a memory of its shape in the previous state, leading to reduced cycle to cycle variability. Based on this preliminary study, using compact model and circuit simulations, VRRAM are proposed for cochlea and convolutional neural network applications, showing good reliability with significant area gain with respect to planar approaches.

Technological details

TEM cross section of our VRRAM is presented in fig.1. A TiN/SiO ${ }_{2}$ double layer is deposited on a W plug, the TiN thickness being $10 \mathrm{~nm}$. Dry etching is used to pattern a cylinder and reveal a TiN liner, used as a bottom electrode (BE). The etching conditions are particularly critical and were optimized to ensure a vertical profile of the top electrode and reduce the corners effects at the $\mathrm{TiN} / \mathrm{SiO}_{2}$ interfaces. NMOS transistor is used as selector of the memory.

\section{VRRAM characteristics}

1. Performances - Typical bipolar IV forming SET and RESET characteristics are showed in fig.2. Low resistance state (LRS) and high resistance state (HRS) resistances versus SET current ( $\mathrm{I}_{\mathrm{SET}}$ ) dependence (fig.3) show an increase of both $R_{L R S}$ and $R_{H R S}$ as $I_{S E T}$ decreases. Functionality down to $7 \mu \mathrm{A}$ - with $\sim 2.5$ decades of mean window margin but higher dispersion - is put in evidence. The higher $\mathrm{R}_{\mathrm{HRS}}$ at low operating currents results in higher $\mathrm{V}_{\mathrm{SET}}$ as seen in fig.4. HRS resistance gradually increases with the RESET voltage as illustrated in fig.5. The forming voltage $\left(\mathrm{V}_{\mathrm{F}}\right)$ is controlled by the Ti top electrode thickness (fig.6), $\mathrm{V}_{\mathrm{F}}$ saturating to $\sim 3.3 \mathrm{~V}$ for $\mathrm{Ti}>30 \mathrm{~nm}$. $200^{\circ} \mathrm{C}$ retention (fig.7) is insured for both LRS and HRS $\left(\mathrm{I}_{\mathrm{SET}}=100 \mu \mathrm{A}\right)$. Switching capability is demonstrated down to $20 \mathrm{~ns}$ at $2.3 \mathrm{~V}$ (fig.8). Endurance performances can be adjusted, playing with the SET and RESET conditions, in order to optimize the window margin, the number of cycles or the SET current (fig.9). At least $10^{7}$ cycles could be achieved with 1 decade of window margin (fig.11).

2. Variability - Resistance and switching voltages variability (including both cell to cell and cycle to cycle contributions) can lead to reliability degradation of RRAM based neural networks, in particular in case of internal switching probability. As expected, the resistance standard deviation increases with the mean value for both HRS and LRS (fig.12) in agreement with [4]. Moreover, an interesting feature concerns the fact that the resistance values during cycling do not follow a pure random process but are correlated from one cycle to the other [5] (fig.13). Correlation (quantified by the correlation coefficient) decreases over cycling after $\sim 50$ cycles (fig. 14). Cycle to cycle correlation means that, despite no resistance drift is measured (fig. 15 bottom), the dispersion of the resistance for a given cycle increases as cycling goes on (fig. 15 top). This means that the conductive filament keeps for $\sim 50$ cycles a "memory" of the shape and resistance it had in the previous cycles. This resistance correlation leads to a correlation of the switching voltage itself, as seen in fig.16. Again, no switching voltage drift is measured but its standard deviation increases during endurance, and saturates after $\sim 30$ cycles (fig. 17). In summary, for applications requiring a limited ( 10) number of cycles, correlation between adjacent cycles reduces variability (fig. 18).

\section{Neuromorphic applications}

1. Synapse behavior implementation - In our approach, a synapse is composed by stacked VRRAM with one common select transistor (Fig.25 left). This offers significant area gain with respect to neural networks in planar configuration with 1T1R elements in parallel (Fig.25 right) [8]. The
OxRAM cells operate in binary mode, only two distinct resistive states (LRS and HRS) per device. The analog-like conductance behaviour is achieved thanks to the parallel of $n$ OxRAM cells. We use the intrinsic variability of RRAM in order to implement progressive on line learning (for instance the stochastic learning rule [3]). The switching probability is governed by the RRAM itself (internal switching probability). Consequently, VRRAM based neural networks (this work) offer area gain due to (1) stacked VRRAM, (2) ITNR configuration and (3) no random number generator circuit. $1^{\text {st }}$, we use VRRAM to emulate the typical progressive behaviour of synapse response. Fig.19 shows the percentage of switched cells ( 50 VRRAM measured) as a function of the applied bias and pulse times. SET and RESET conditions can be identified to control the probability to switch the memory with a given value for each pulse, the probability being imposed by the application and neural network structure. An OXRAM compact model [6] is then used to set a model card based on our experimental results. Fig.19 is fitted with good agreement, while cell-to-cell and cycle-to-cycle variability is well reproduced (fig.20). Then the model is used to simulate a synapse composed by stacked VRRAM with 2 to 24 levels (fig.23). The VRRAM are addressed in the same time (one selector for one VRRAM pillar), and the output signal corresponds to the sum of all the VRRAM in parallel (fig.22). Then we calculated the standard deviation of the number or required pulses to SET or RESET half of the VRRAM stacked in one pillar (fig.23). Fig.24 shows that $\sigma$ decreases as the number of levels increases. Thus it is possible to identify the required number of levels to reach a given $\sigma$ and emulate typical analogic synaptic behaviour.

2. Cochlea - In the case of real-time auditory pattern extraction (inspired from a 64-channel silicon cochlea emulator, figs.25-26) [3] at least 3 RRAM are required per synapse [4]. In our case, one synapse is thus composed by 3 stacked VRRAM with one select transistor, offering an integration density improvement of $>3$ with respect to previous work [4]. Pulse times and voltages are adjusted based on fig. 19 in order to target switching percentages of $20 \%$ for SET and $5 \%$ for RESET [7]. As the number of required cycles in cochlea application is high $\left(>10^{5}\right)$ [7], both cell-to-cell and cycle-to-cycle variability are taken into account and affect the internal switching probability. Nevertheless, the circuit behaviour shows good agreement with respect to the perfect case involving an external random number generator (fig.27). Finally, we also assumed a perfectly controlled VRRAM technology where only cycle-to-cycle variability leads to switching voltage dispersion $(\sigma / 3$ in fig. 18$)$. In this configuration, very similar results to the perfect case are obtained (fig.28).

3. Convolutional Neural Network (CNN) - VRRAM can also be a suitable solution for visual pattern extraction applications (inspired from the processing inside visual cortex). It has been demonstrated that for Convolutional Neural Networks (CNN) a higher VRRAM density per synapse is required $(\sim 20)$ [8]. Based on the design used for the recognition of handwritten digits shown in fig.29 and thoroughly described in [4] it appears (from simulations, fig.30) that given a certain resistance distribution (fig.18 left) the recognition rate depends on the number of stacked VRRAM cells. Thus, at least 12 VRRAM levels are required to reach $>98 \%$ circuit reliability. As CNN applications require limited number of cycles, correlation reduces RRAM cycle to cycle variability and improves circuit reliability with internal switching probability. Being able to stack several memory cells on a pillar (VRRAM) a considerable gain in total area (x10) is obtained compared to a standard design using planar devices. Further gain could even be envisaged for more complex applications requiring more synaptic levels.

\section{Conclusions}

In this paper, vertical RRAM are investigated and proposed to increase the density of neuromorphic circuits, one 1TnR pillar emulating one synapse. For cochlea application, good agreement with planar binary OXRAM configuration with random generator is obtained, with an area gain of more than a factor 3. Resistance correlation between adjacent cycles improves the reliability for neuromorphic applications requiring low endurance performances. Thus, for convolutional neural network, 10-15 RRAM levels are sufficient to reach a recognition rate of more than $98 \%$. VRRAM based synapses open the path to high density neuromorphic circuits requiring aggressive synaptic levels. 
References

[1] I. G. Baek et al., 2011 IEDM Tech. Dig. pp.737-740.

[2] I. T. Wang et al., 2014 IEDM Tech. Dig., pp.665-668.

[3] M. Suri et al., IEDM 2012 Tech. Dig., pp.235-238.

[4] D. Garbin et al., IEDM 2014 Tech. Dig., pp. 661-664.

[5] A. Calderoni et al., proc. of IMW 2014.

[6] M. Bocquet et al., IEEE Trans. on Elec. Dev. 61, 3, 674-681, 2014

[7] M. Suri et al., IEEE Trans. on Elec. Dev. 60, 7, 2402-2409, 2013.

[8] D. Garbin et al., IEEE Trans. on Elec. Dev. , 2015.

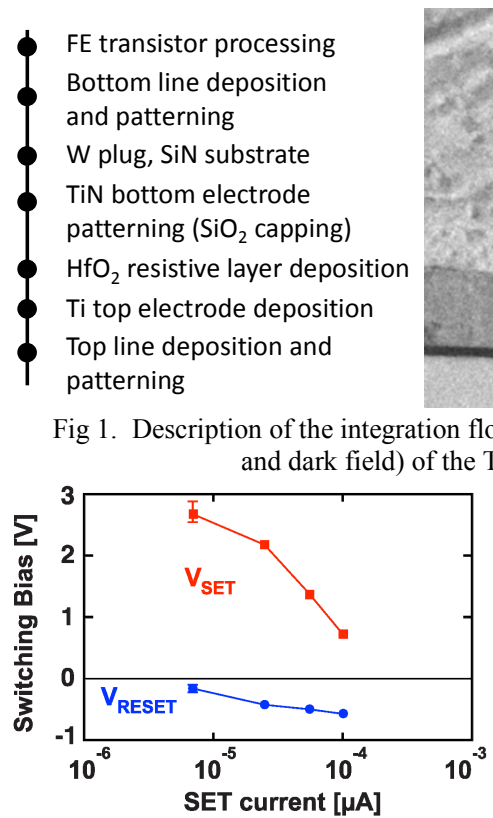

Fig. $4 \mathrm{~V}_{\mathrm{SET}}$ and $\mathrm{V}_{\text {RESET }}$ as a function of the SET current. Higher $\mathrm{V}_{\mathrm{SET}}$ is due to

Fig. 3 LRS and HRS resistances as a function of the SET current.

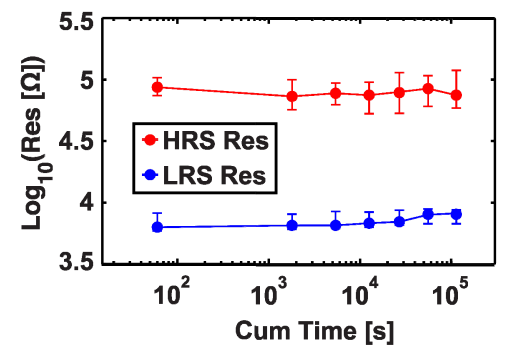

Fig. $7200^{\circ} \mathrm{C}$ HRS and LRS retention. Median and percentiles (30\%-70\%) are represented.

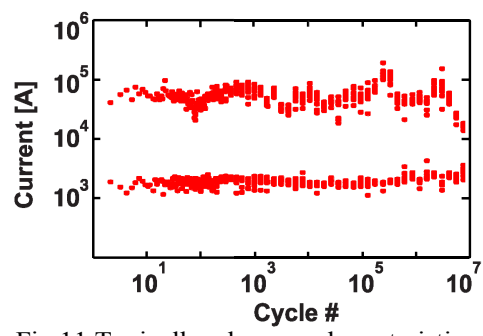

Fig.11 Typicall endurance characteristics targetting a high number of achieved
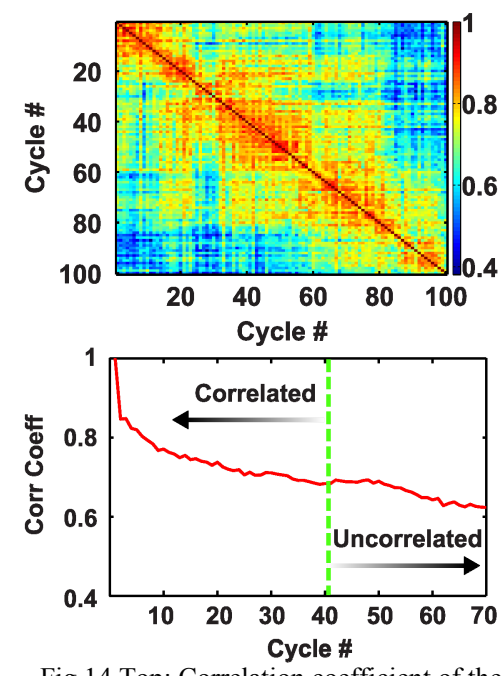

Fig.14 Top: Correlation coefficient of the cell resistance for 50 cells over 100 cycles. Bottom: 1D cut of the curve.

$$
\text { cycle. }
$$
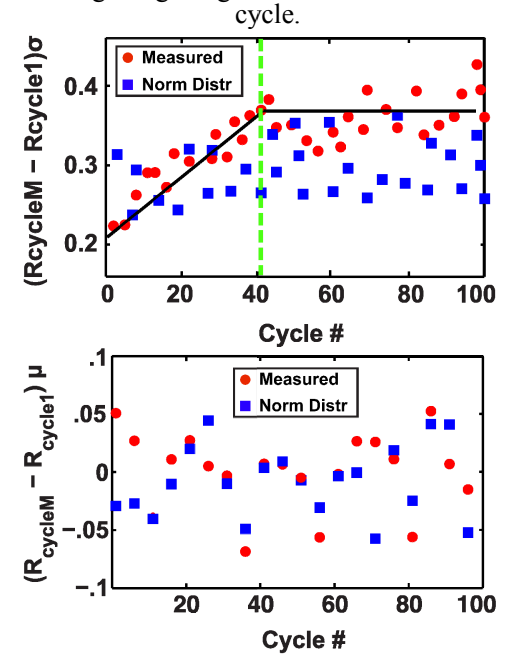

Fig.15 Top: Dispersion of the cell resistance shift during cycling. Bottom: Cell resistance drift during cycling.

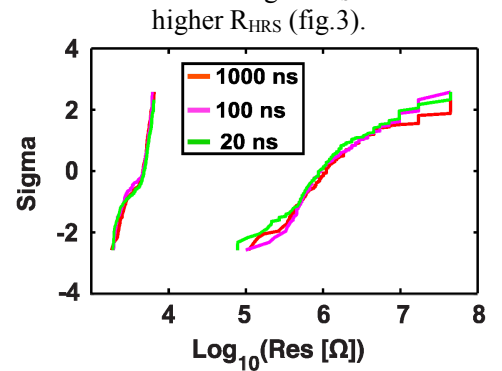

Fig.8 LRS and HRS distributions for various programming pulse widths (20ns $1 \mu \mathrm{s})$ for $\mathrm{V}_{\text {pulse }}=2.3 \mathrm{~V}$.

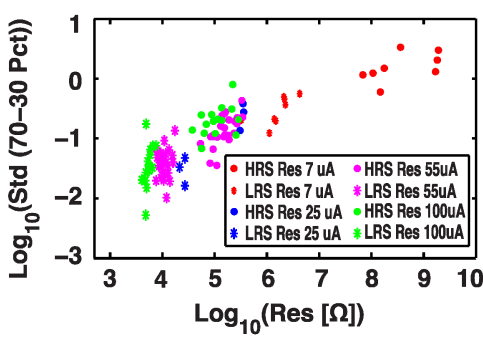

Fig. 12 LRS and HRS resistance dispersion as a function of the resistance median.
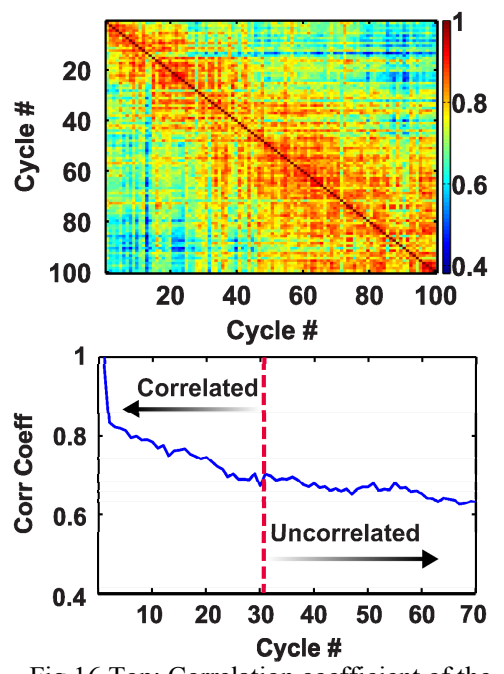
cell switching voltage for 50 cells over 100 cycles. Bottom: 1D cut of the curve.
Fig.16 Top: Correlation coefficient of the

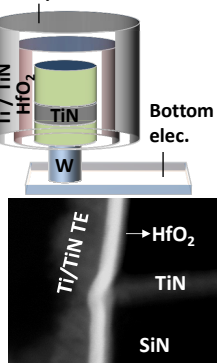

SiN

w, and TEM cross sections (high resolution

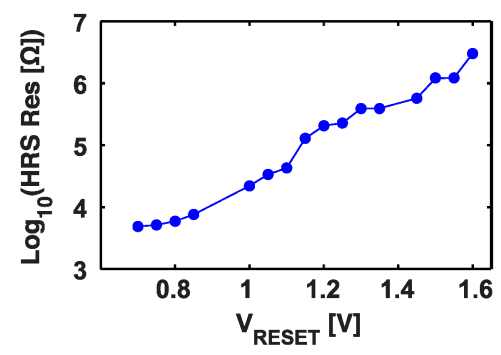

Fig.5 HRS resistance as a function of the bit line RESET voltage.

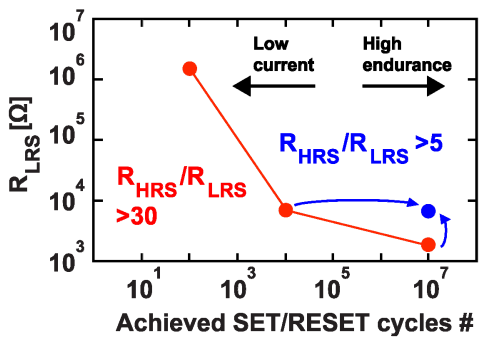

Fig.9 Relation between LRS resistance

(related to operating current) and achieved number of cycles during endurance.

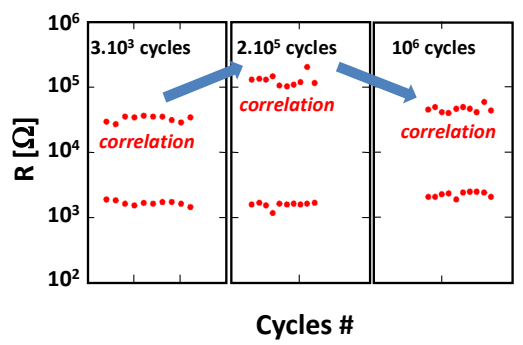

Fig.13 Zoom on cell endurance (fig.11) showing resistance correlation between adjacent cycles.
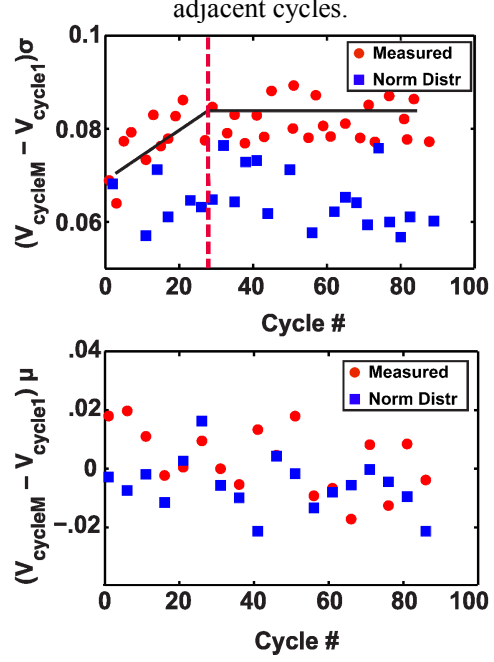

Fig.17 Top: Dispersion of the switching voltage shift during cycling. Bottom: Switching voltage drift during cycling. 
